\title{
Estrategia de evaluación basada en juegos: Caso Ingeniería de Sistemas Universidad de Medellín
}

\author{
Games-based assessment strategy: Case Systems Engineer of Universidad de Medellín \\ María Clara Gómez-Álvarez ${ }^{1 *} \quad$ Jaime Alberto Echeverri ${ }^{1} \quad$ Liliana González-Palacio $^{1}$ \\ Recibido 19 de abril de 2016, aceptado 28 de noviembre de 2016 \\ Received: April 21, $2016 \quad$ Accepted: November 28, 2016
}

\begin{abstract}
RESUMEN
La evaluación es un elemento fundamental del proceso de enseñanza-aprendizaje, ya que permite valorar la evolución de los estudiantes. Las nuevas reglamentaciones del Ministerio de Educación Nacional de Colombia a este respecto tienen como finalidad la evaluación formativa, integral y cualitativa, centrada en el desarrollo de las habilidades de los estudiantes más que en los contenidos de enseñanza. Estos cambios en la concepción de la educación exigen nuevas estrategias para motivar al estudiante y para propiciar espacios que disminuyan el estrés que generan las actividades evaluativas. El presente trabajo describe los resultados de la utilización de un videojuego/juego de computadora como actividad evaluativa en una asignatura del programa de pregrado en Ingeniería de Sistemas de la Universidad de Medellín. Estos resultados demuestran que la incorporación de videojuegos/juegos de computadora en el proceso evaluativo fomenta el trabajo en equipo y un mayor compromiso de los estudiantes frente a su proceso de aprendizaje.
\end{abstract}

Palabras clave: Evaluación formativa, motivación, proceso de enseñanza-aprendizaje, juegos de computador.

\begin{abstract}
Assessment is a key element of the teaching-learning process as it allows monitoring of student's progress. New regulations emanating from the Colombian Ministry of Education look for the promotion of formative, integral and qualitative assessment practices targeting the development of student's abilities rather than mere acquisition of course content. Meeting these new goals requires innovative strategies to motivate students while reducing the stress generated by assessment activities. This paper describes the results of the introduction of a videogame /computer's game as an evaluative activity in a course of the System Engineering undergraduate program at Universidad de Medellin. These results reveal that the incorporation of computer's games in assessment activities encourages teamwork and promote greater students' involvement in the learning process.
\end{abstract}

Keywords: Formative assessment, motivation, stress reduction, teaching-learning process, computer games.

\section{INTRODUCCIÓN}

La preocupante tasa de fracaso universitario actual, reflejada en los altos índices de deserción académica depende de diferentes factores como falta de pers- pectivas de éxito, carencia de alicientes para el estudio, apatía escolar, desesperanza constante, disminución de la propia autoestima, la "falta de ganas de aprender". Estos factores son incuestionables y de gran peso en la realidad educativa a todos los

1 Facultad de ingeniería. Universidad de Medellín. Carrera 87 No 30-65 Medellín, Colombia. E-mail: mcgomez@udem.edu.co; jaecheverri@udem.edu.co; ligonzalez@udem.edu.co

* Autor de correspondencia. 
niveles en Colombia. El tema se torna crítico en lo relacionado con el desarrollo profesional de los jóvenes que ingresan a instituciones de educación superior [1]. Pérez-Gama y otros [2], afirman que los factores que impactan la deserción en la educación superior en Colombia son: i) estrato socioeconómico, ii) pobre desempeño académico en el colegio, iii) falta de orientación vocacional en la formación media, y iv) falta de adaptación al ambiente universitario.

Por su parte, López y otros [3] resaltan algunos aspectos importantes en el fenómeno de deserción en programas de ingeniería en Colombia, como son: i) la mayor tasa de deserción se concentra en el primer año de programas de ingeniería, ii) la necesidad de acciones concretas para mejorar el desempeño de los estudiantes en áreas de fundamentación como matemáticas y comprensión lectora, y iii) la influencia de la diversidad y heterogeneidad de estudiantes en la deserción.

El proceso de enseñanza-aprendizaje es una labor compleja que puede suponer grandes desafíos en los estudiantes y docentes, ya que solicita un gran esfuerzo por parte de ambos. De acuerdo con Michalko [4], involucrar dinámicamente a los estudiantes en las áreas de conocimiento por medio de herramientas no tradicionales de enseñanza-aprendizaje incrementa su capacidad de abstracción, facilita la profundización y desarrolla el pensamiento crítico así como la habilidad de solucionar problemas cotidianos. La posibilidad de recibir información inmediata sobre la precisión de las respuestas es un aspecto que anima y estimula en los procesos cognitivos [5], el trabajo colaborativo entre los participantes por medio de juegos y su interrelación mejora el proceso de aprendizaje y se convierte en una herramienta poderosa para facilitar un aprendizaje significativo [6].

Por otro lado, los juegos de computador gozan de gran popularidad entre jóvenes en la actualidad, la capacidad de las nuevas generaciones para el manejo de las tecnologías modernas es evidente y un buen enfoque en la aplicación de estos como estrategia de aprendizaje puede producir excelentes resultados en los procesos cognitivos de nuestros adolescentes. Además es una gran oportunidad para aumentar los niveles de motivación, componente esencial del proceso de enseñanza-aprendizaje [7].
En el programa de Ingeniería de Sistemas de la Universidad de Medellín se viene trabajando en la dinamización del proceso de enseñanza-aprendizaje en el aula por medio de la aplicación de juegos. El objetivo es generar motivación, participación y mejor asimilación de conceptos por parte de los estudiantes. Se busca además el desarrollo de un conjunto de competencias requeridas por cualquier ingeniero, para garantizar que la solución a problemas planteados incorpore innovación.

En este artículo se presenta el juego "Guerra de estrellas" como una alternativa para evaluar temáticas de una asignatura de la línea de Ingeniería de Software. El propósito es mantener altos niveles de motivación en los estudiantes y minimizar los factores como el estrés que afectan el desempeño. Adicionalmente, este juego es desarrollado como proyecto de aula, en dos asignaturas de programación. Esto se convierte en un incentivo adicional para los estudiantes, por el reconocimiento asociado a que su aplicación sea la elegida para aplicar en otra asignatura del programa de Ingeniería de Sistemas.

La estructura del presente artículo es la siguiente: en la sección de marco teórico se presentan los conceptos fundamentales que sustentan esta propuesta; posteriormente se describen algunos trabajos relacionados con el proceso de evaluación de estudiantes; después se plantea el diseño de la estrategia de evaluación basada en juegos y los resultados obtenidos. Finalmente se presentan las conclusiones y el trabajo futuro.

\section{MARCO TEÓRICO}

La evaluación es considerada uno de los componentes principales del proceso de enseñanza-aprendizaje [8]. Autores como Alart [9] definen la evaluación como un proceso participativo para medir la evolución del aprendizaje del estudiante y mejorar el diseño y desarrollo de la práctica docente. Por su parte, Ortiz [10] plantea que la evaluación se ocupa de valorar los cambios generados en el conocimiento de un estudiante sobre un tema específico a partir de la revisión de la información adquirida de forma sistemática, gradual y continua.

En el caso de un programa de ingeniería de sistemas, es fundamental el desarrollo de competencias como capacidad de abstracción, razonamiento lógico o 
pensamiento sistémico, entre otras. Por esto se hace necesario aplicar estrategias de evaluación tradicionales (exámenes, talleres o prácticas). También se debe acudir a estrategias que permitan a los estudiantes participar activamente en el proceso de enseñanza-aprendizaje y en su evaluación [11].

Los juegos son una estrategia de enseñanza que permite a los estudiantes aprender haciendo, acelerar el aprendizaje y mejorar la recordación de conceptos [12]. Estas ventajas, sumadas al factor motivacional, buscan generar mejores resultados de los estudiantes al abordar las actividades evaluativas de las diferentes asignaturas. Frente al proceso de evaluación, el docente tiene la importante responsabilidad de seleccionar técnicas adecuadas. Se trata de medir el logro de competencias y generar un ambiente de confianza donde el estudiante evidencie su aprendizaje minimizando situaciones de tensión y frustración. La utilización de juegos para evaluar ofrece: interacción constante, sentimiento permanente de desafío y competencia para lograr un mayor nivel de involucramiento, combinación entre diversión y aprendizaje significativo.

\section{TRABAJO RELACIONADO}

En esta sección se plantean dos líneas de revisión: la primera orientada hacia la identificación de los diferentes tipos de evaluación existentes; la segunda línea explora el uso de los juegos durante el proceso de enseñanza-aprendizaje.

Con respecto a la primera línea, González y otros [13] proponen la siguiente taxonomía:

- Enfoque tradicional: de tipo cuantitativo y memorístico.

- Enfoque conductista: se realizan pruebas de tipo repetitivo y cuantitativo como exámenes de selección múltiple, preguntas de falso/verdadero, buscando sistematizar el conocimiento asimilado por el estudiante y la verificación del logro de objetivos de aprendizaje.

- Enfoque contemporáneo: basado en un proceso donde el docente observa, analiza, identifica, valora, diferencia, presenta alternativas, comprueba y toma decisiones. En este caso la evaluación incluye el evento evaluativo (examen, exposición, práctica) y la observación permanente por parte del docente para detectar la evolución del estudiante en términos de conocimientos, actitudes y valores.

- Enfoque social: evaluación dinámica de doble vía donde el docente recopila información por diferentes formas para elaborar juicios, mientras el estudiante es consciente de su proceso de formación. Es de tipo cualitativo y se puede llevar a cabo en forma individual o grupal.

Por otro lado, Ortiz [10] propone los siguientes tipos de evaluación: unidimensional, multidimensional, diagnóstica, formativa, sumativa, reconstructiva, sistemática, basada en paradigmas socioculturales, desde paradigmas complejos y comprensivos. En esta clasificación el autor tiene en cuenta varias dimensiones como: i) las técnicas usadas, ii) el momento donde se aplican, y iii) las responsabilidades asignadas a cada rol involucrado en el proceso evaluativo.

En cuanto a la segunda línea de revisión sobre la utilización de juegos en el proceso de enseñanzaaprendizaje se encuentran diversas propuestas.

Algunos autores usan los juegos como estrategia de enseñanza, es decir, buscando entrenar a los estudiantes en la solución de un determinado problema, en el conocimiento de un determinado contenido o en la mejora de una o varias competencias personales [13-18].

Otras propuestas se orientan a la utilización de juegos en el proceso de evaluación de estudiantes, aunque en un porcentaje menor con respecto al uso de juegos para enseñar conceptos. Existen iniciativas donde se incorporan simulacros, actividades previas de motivación y propuestas más elaboradas como la formulación de evaluaciones según el estilo cognitivo de los estudiantes, o aplicar pruebas de figuras enmascaradas (EFT Embedded Figures Test) [17].

Algunas aproximaciones se centran en aplicar "juegos de torneo" para las evaluaciones. Se trata de una estrategia de aprendizaje puramente constructivista y cooperativo [20]. Consiste en sustituir la actividad de evaluación por un torneo de preguntas en el que participan los estudiantes agrupados en equipos de 4 o 5 , estructurados según el rendimiento académico previo de los estudiantes y que compiten segregados en distintas mesas según ese nivel de rendimiento [20]. 
Otros autores [16] proponen abordar la evaluación como un proceso experimental de validación de conocimientos en el área de interés por medio de reuniones presenciales y el uso de herramientas web 2.0.

En este mismo sentido Watkins [21] plantea usar la web 2.0 para evaluación por pares mediante el desarrollo de proyectos grupales donde cada estudiante evalué el conocimiento adquirido por los demás miembros de su grupo de trabajo.

En el presente artículo se describe una estrategia de incorporación de juegos como actividad evaluativa de una asignatura del programa de ingeniería de sistemas. En esta propuesta se busca usar un enfoque orientado por procesos donde el producto entregado por los estudiantes en los proyectos de aula de asignaturas de primeros semestres (juegos) sirve como insumo de evaluación en otra asignatura de nivel más avanzado.

\section{DISEÑO DE LA ESTRATEGIA}

En esta sección se explica la construcción y posterior uso del juego "Guerra de estrellas" como medio para aplicar una evaluación intermedia en el curso "Ingeniería de software I". Se buscó incorporar lúdicas y conceptos que facilitan un proceso colaborativo y multidimensional con enfoque social para la evaluación [19].

El desarrollo del juego se realizó en dos asignaturas pertenecientes al programa de ingeniería de sistemas de la Universidad de Medellín: Programación Orientada a Objetos-POO- y Lenguajes de programación. En POO, materia de primer semestre, los estudiantes aprenden los conceptos de clase y de objeto, y las interacciones entre ellos, con el propósito de construir programas siguiendo los principios de este paradigma. También se abordan los conceptos de herencia, abstracción, polimorfismo y encapsulamiento. La elección del paradigma de orientación a objetos para el desarrollo del juego se debe a la necesidad de la construcción de elementos que garanticen una solución por medio de la aplicación de los fundamentos del reúso, mantenimiento, la modificabilidad y fiabilidad de la solución planteada.

El curso de lenguajes de programación tiene como objetivo darle continuidad a POO al aplicar conceptos en el desarrollo de una herramienta didáctica diseñada con las directrices del docente. La Figura 1 presenta las metas de la aplicación del juego como ejercicio de clase en la interacción entre ambas asignaturas. Adicionalmente, permite comprender las metas de cada una de las etapas del proceso, y como se articulan las dos asignaturas, tanto en el desarrollo del juego, y su uso como herramienta de evaluación.

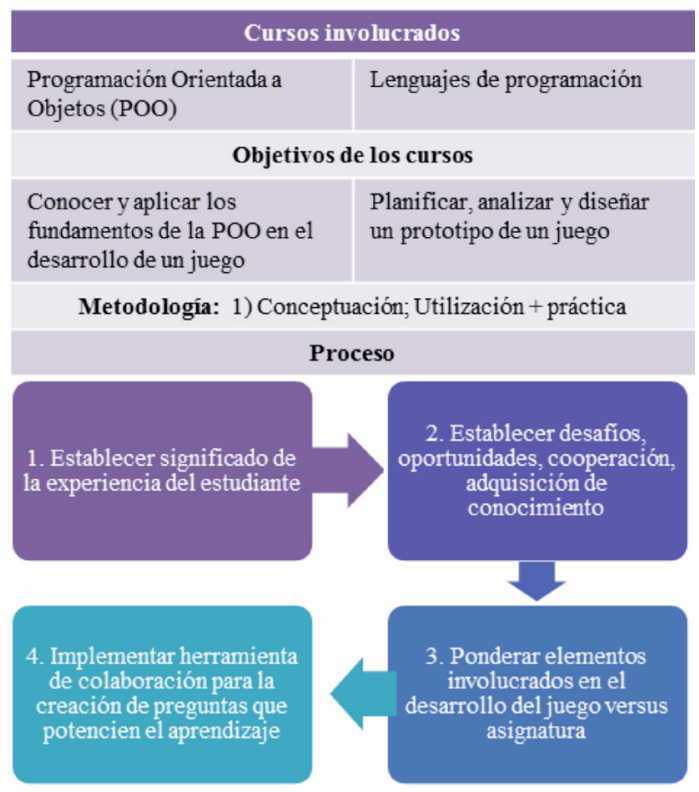

Figura 1. Metas/etapas en la construcción del juego.

Para la ejecución del juego se eligieron preguntas de selección múltiple con única respuesta por ser ideales si se desea incorporar conceptos de una temática específica y proveer interrogantes que se puedan contestar de manera rápida (limitada por espacios de tiempo predefinidos). Los tiempos cortos buscan mantener la atención y concentración en el juego, generados por la competencia y el desafío entre equipos de estudiantes.

Por otra parte, la selección de guerra de estrellas como lúdica, estuvo enfocada a la posibilidad de contar con estrategias, como la mejor ruta de acuerdo a las celdas ganadas por el rival o celdas propias. Un juego que permitiera mantener la atención de los estudiantes durante su ejecución, donde el tablero de juego se convierte en el escenario principal, y cada equipo dispone de diferentes caminos, tanto para bloquear, como para ganar. 
De la misma manera los diferentes niveles en el juego permiten evolucionar en el conocimiento de un tema específico de la asignatura, convirtiendo el juego en un desafío entre equipos de estudiantes, lo que permite la estimulación por el conocimiento del tema que se evalúa.

Las preguntas fueron discriminadas en cinco niveles de acuerdo al grado de dificultad y atendiendo la taxonomía de Bloom. Es así como el primer nivel se enfoca en evaluar habilidades de identificación, clasificación, reconocimiento. Las preguntas del nivel dos buscan medir habilidades de comprensión útiles para describir, comparar, convertir, jerarquizar. Las de nivel tres en habilidades de aplicación útiles para emplear elementos, construir prototipos, estructurar módulos de programación y funcionalidades. Las preguntas nivel cuatro buscan medir el componente analítico en los jugadores (estudiantes), habilidades relacionadas con la diagramación, abstracción. Las preguntas del último nivel buscar medir la capacidad para planear, construir, generalizar, contrastar y verificar por medio de pruebas. El tablero en el juego permite a los jugadores tomar decisiones que pueden ser favorables (o desfavorables) para vencer al equipo oponente.

En este caso se construyó la denominada "Guerra de estrellas" donde dos participantes (o grupos de participantes) compiten entre sí, buscando formar un camino conexo entre un punto de partida y uno de llegada. La Figura 2 muestra la interfaz.

Como plataforma de desarrollo se utilizó Visual Studio.Net, entorno de desarrollo integrado (IDE) para sistemas operativos Windows, con soporte para C++, C\#, Visual Basic .NET, F\#, Java, y entornos de desarrollo Web. Como lenguaje se trabajó C\# (o C-Sharp) que es completamente orientado a objetos, que permite definir una o más clases dentro de un mismo espacio de nombres y facilita un rango amplio de tipos de datos. El diseño del juego (Figura 1), tiene un espacio para las preguntas y respuestas, y un tablero de juego. Cuando el jugador selecciona una casilla aparece una pregunta (seleccionada al azar), el sistema advierte si la respuesta fue acertada, en cuyo caso la casilla seleccionada quedará con un color que identifica al equipo, si la respuesta fue incorrecta entonces la casilla queda con el color inicial y sigue disponible para otra jugada de cualquier equipo.

Al inicio del juego todas las preguntas son etiquetadas como disponibles. Una vez una pregunta es seleccionada en la base datos, y se responde correctamente es marcada como no disponible para evitar repetición de preguntas. Los jugadores (o equipos) pueden seleccionar entre cinco niveles de dificultad de las preguntas, lo que hace más interesante el desafío y la competencia, creando diversión e interacción constante entre los grupos de estudiantes.

Las respuestas de cada jugador (equipo) se marcan en los colores rojo o azul. El jugador o equipo que primero logre formar un camino conexo entre las columnas de la matriz de juego (de izquierda a derecha o viceversa) será el vencedor.

El juego fue aplicado reemplazando un evento evaluativo individual sobre técnicas de educción de requisitos como parte del proceso de ingeniería de software. El examen escrito tenía 30 preguntas de selección múltiple. Algunos ejemplos de preguntas se presentan a continuación:

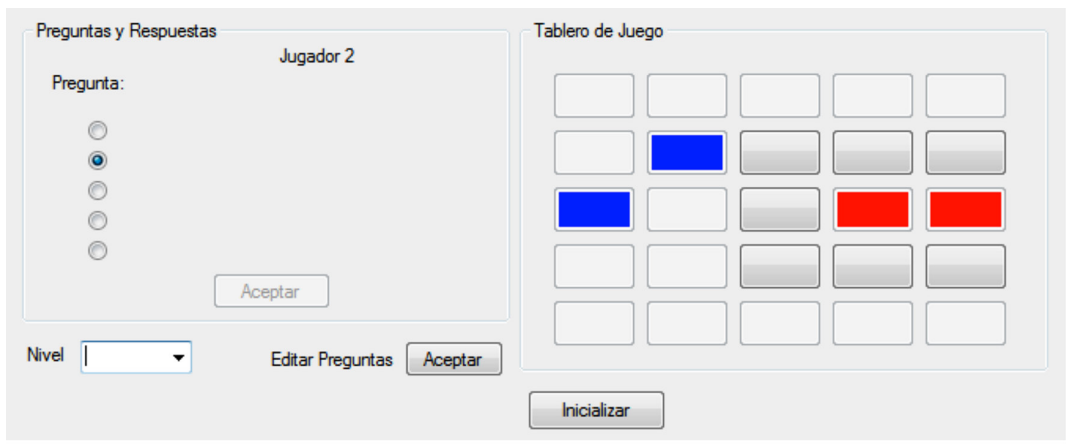

Figura 2. Tablero de juego. 
Pregunta: En la técnica tormenta de ideas:

a) Las ideas que se expongan deben ser criticadas directa o indirectamente.

b) Los participantes obtienen información detallada de un individuo.

c) Está permitida la crítica y el debate.

d) Los participantes exponen sus puntos de vista sin restricciones.

Pregunta: La técnica de observación o etnografía propone que:

a) Los ingenieros de software aprendan sobre las tareas del usuario por medio de la inmersión dentro de su ambiente laboral.

b) Los ingenieros de software aprendan sobre las tareas del usuario leyendo manuales y documentación.

c) Los ingenieros de software aprendan sobre las tareas del usuario buscando sistemas similares en internet.

d) Los ingenieros de software aprendan sobre las tareas del usuario entrevistándolos.

Estas mismas preguntas fueron ingresadas al juego "Guerra de estrellas" para el semestre 2015-01 y se probó el efecto de resolverlas con la dinámica del juego, en lugar de hacerlo de forma individual. En la Figura 3 se muestran evidencias.

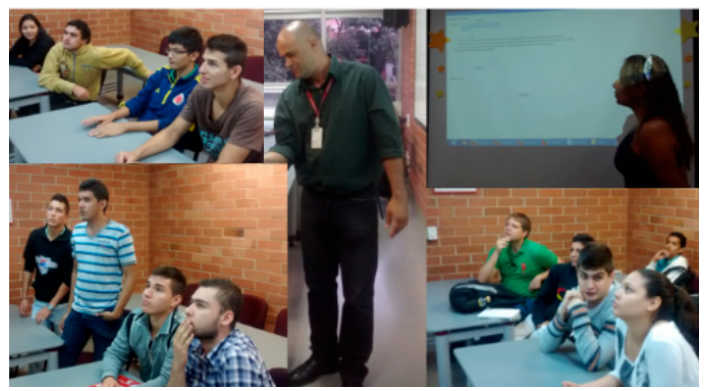

Figura 3. Evidencias al aplicar "Guerra de estrellas".

En resumen, para el segundo semestre de 2014 se ejecutó una prueba escrita sobre técnicas de educción de requisitos. En el primer semestre de 2015 se utilizó el juego para evaluar la misma temática.

A continuación, se enuncian algunos controles usados para evitar sesgos:
- Los dos grupos involucrados (en 2014 y 2015) fueron orientados por un solo profesor que dictó los mismos contenidos (nombre del curso: software 1).

- Los rangos de edad en ambos grupos oscilan entre los 17-20 años.

- El tema para evaluar fue el mismo en ambos grupos: técnicas para captura o educción de requisitos.

- Para ejecutar el juego se conformaron equipos de 2 o 3 estudiantes seleccionados aleatoriamente. Todos los estudiantes del curso vivieron la experiencia de jugar y competir con otros equipos.

- El grupo que hizo la evaluación escrita convencional fue de 16 estudiantes. El curso donde se evaluó mediante el juego tuvo un tamaño de 15 estudiantes.

\section{RESULTADOS}

Los resultados del experimento son presentados en esta sección. El análisis se hace desde dos puntos de vista: i) desempeño de los estudiantes en términos de la nota obtenida en la actividad evaluativa y ii) percepción global de los estudiantes sobre el uso de lúdicas para evaluar su proceso de aprendizaje.

Frente a la valoración del desempeño de los estudiantes se realizó una comparación de las notas definitivas de la misma actividad evaluativa (en términos de contenido) con dos estrategias distintas (examen escrito vs juego guerra de estrellas) de dos grupos diferentes de la asignatura Ingeniería de Software I (ver Tabla 1). En ambas actividades se

Tabla 1. Comparación de notas definitivas de estudiantes con examen escrito vs. juego "Guerra de estrellas" para Ingeniería de Software I.

\begin{tabular}{|c|c|c|c|}
\hline Grupo & $\begin{array}{c}\text { Estrategia } \\
\text { de } \\
\text { evaluación }\end{array}$ & $\begin{array}{c}\text { Nota } \\
\text { promedio }\end{array}$ & Varianza \\
\hline $\begin{array}{c}\text { Grupo A } \\
\text { Semestre } \\
02-2014\end{array}$ & $\begin{array}{c}\text { Examen } \\
\text { escrito }\end{array}$ & 3,31 & 0,625 \\
\hline $\begin{array}{c}\text { Grupo B } \\
\text { Semestre } \\
01-2015\end{array}$ & $\begin{array}{c}\text { Juego } \\
\text { "Guerra de } \\
\text { estrellas" }\end{array}$ & 3,75 & 0,61 \\
\hline
\end{tabular}


pretendió mantener la coherencia entre el objetivo de evaluación y el instrumento seleccionado, también se buscó medir por medio de ejercicios prácticos la asimilación de los conceptos necesarios para comprender, teniendo presente que en ambas actividades los contenidos de las pruebas fueron exactamente los mismos. Las evaluaciones efectuadas estuvieron centradas en el aspecto formativo. Se buscaba evaluar el progreso del estudiante durante el semestre, también medir el logro de habilidades específicas definidas en el microcurrículo de las asignaturas en cuestión.

A partir de los resultados observados en la Tabla 1 se concluye que los resultados individuales de cada estudiante se encuentran relativamente concentrados en torno a la media para ambos grupos, es posible inferir que hubo una mejora de aproximadamente 9\% cuando se empleó la evaluación por medio de lúdicas. Este incremento da una idea del potencial de los juegos como estrategia evaluativa, siendo una motivación para continuar en esta línea de trabajo y pensar en otro tipo de juegos para incorporar al proceso evaluativo en el programa de Ingeniería de Sistemas. Cabe aclarar que este es un piloto y para validar completamente los hallazgos sería necesario aplicar el mismo experimento a una muestra más representativa.

Por otro lado, con el propósito de conocer la percepción de los estudiantes frente a la utilización de lúdicas para evaluar su desempeño al finalizar el curso se les aplicó una encuesta anónima. Algunas preguntas se presentan a continuación:

1. A su criterio ¿qué competencias se fortalecieron con la actividad evaluativa "Guerra de estrellas"?

2. Considera que las evaluaciones apoyadas por juegos:

a) Disminuyen la tensión frente a la actividad.

b) Aumentan la tensión frente a la actividad.

c) Le es indiferente.

3. Frente al nivel de recordación de conceptos, consideras que las evaluaciones mediante juegos:

a) Aumentan el nivel de recordación.

b) Disminuyen el nivel de recordación.

c) No influyen en el nivel de recordación.

4. Prefieres que las evaluaciones se realicen:

a) Con el esquema tradicional (examen escrito).

b) Mediante el uso de plataformas de e-learning.

c) Mediante lúdicas. d) En modalidad mixta (combinación de las opciones anteriores).

La pregunta 1 se orienta a las competencias que los estudiantes consideran se promovieron durante la actividad: $35 \%$ - Refuerzo de conceptos y $65 \%$ Trabajo en equipo. Estos porcentajes evidencian el hecho de que una de las competencias a las que se enfocan los videojuegos en el proceso de formación es la promoción del trabajo en equipo.

En lo relacionado con la tensión que generan las actividades evaluativas en los estudiantes, las respuestas a la pregunta 2 (Figura 4) muestran que la mayoría de ellos (80\%) coincide en que las evaluaciones basadas en juegos disminuyen el nivel de tensión de los estudiantes lo que está alineado con uno de los grandes retos de evaluación de estudiantes: combinar entorno agradable y preguntas que permitan determinar el conocimiento de los conceptos técnicos de una temática. Este es uno de los resultados más importantes de esta prueba piloto, ya que muestra de manera contundente que los estudiantes prefieren actividades evaluativas

\section{Consideras que las evaluaciones apoyadas por juegos}

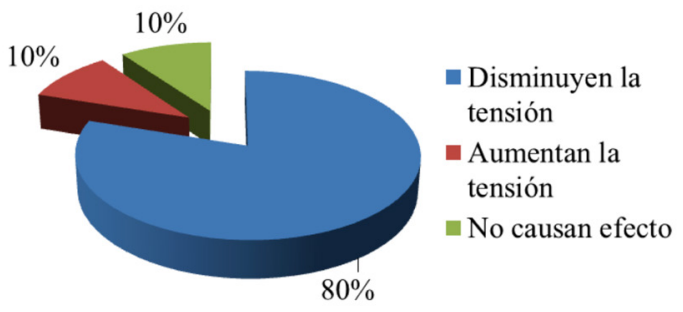

Frente al nivel de recordación, consideras que las evaluaciones mediante juegos:

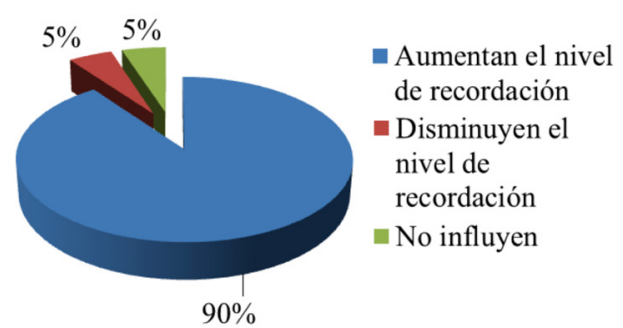

Figura 4. Respuestas de los estudiantes frente a su valoración de evaluaciones realizadas con juegos vs. tensión y nivel de recordación. 
donde no solo se valore su nivel de conocimiento, sino que se cuente con un ambiente agradable para su realización.

Diversos autores enuncian el nivel de recordación de conceptos como una de las ventajas cuando se utilizan juegos en el proceso de enseñanza [5]. Esto también se observa en el proceso de evaluación apoyado en juegos según la pregunta 3 , donde el $90 \%$ de los estudiantes consideran que los juegos para evaluar aumentan el nivel de recordación de conceptos. Esto se debe a que la aplicación del juego en repetidas ocasiones en el salón de clase, con la motivación de una nota o bonificación, hace que el estudiante esté atento y se esmere en asociar conceptos para cuando sea su turno.

Finalmente, en la Figura 5 se observan las preferencias de los estudiantes frente a las modalidades de evaluación disponibles: $55 \%$ - Modalidad mixta, 40\% - Mediante lúdicas, $5 \%$ - Mediante herramientas de e-learning y $0 \%$ - Evaluación escrita. Estas cifran confirman una inclinación de los estudiantes por formas de evaluar apoyadas en lúdicas (40\%) o estrategias de evaluación que combinen actividades tradicionales como exámenes escritos con actividades en una plataforma de e-learning (55\%). Estos resultados reflejan el deseo de los estudiantes de que los docentes incorporen en el aula de clase las tecnologías de información con las que ellos están tan familiarizados. Igualmente, los estudiantes esperan que los docentes sean creativos e innovadores en las actividades propuestas en clase, incluyendo los eventos evaluativos. Por último, estas respuestas dan cuenta de un llamado

\section{Prefieres que las evaluaciones se realicen}

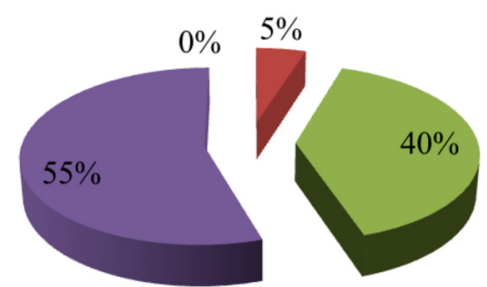

- Forma escrita $\square$ Mediante herramientas e-learning - Con Lúdicas Modalidad mixta

Figura 5. Respuestas de los estudiantes frente a la modalidad de evaluación preferida. permanente de los estudiantes a que los docentes exploremos formas de evaluar que combinen motivación y valoración del aprendizaje significativo buscando evitar que factores como el estrés afecten su desempeño durante la ejecución de actividades evaluativas.

\section{CONCLUSIONES Y TRABAJO FUTURO}

La capacidad de crear ambientes que propicien la motivación de los estudiantes se convierte en una tarea fundamental para los docentes que desean generar competencias en sus estudiantes. En este sentido, los juegos de computador se vislumbran como potenciales aliados en esta búsqueda para facilitar altos niveles de interacción mediante estrategias nuevas. Se logra además desarrollar habilidades como la creatividad, toma de decisiones, capacidad de negociación, liderazgo, buena comunicación y trabajo en equipo.

Vale la pena también analizar los logros obtenidos en cada curso involucrado desde la construcción del juego hasta su uso:

- En los cursos de POO y programación (allí se desarrolló y codificó el juego): se fortaleció la capacidad para comprender, adaptar y asimilar conceptos de la POO como el polimorfismo, encapsulación, herencia y abstracción.

- En el curso de ingeniería de software (allí se usó el juego como medio para evaluar un tema): se logró constatar que las evaluaciones basadas en juegos disminuyen el nivel de tensión de los estudiantes. Además, se combinó un entorno agradable con preguntas que permitan determinar el conocimiento de los conceptos técnicos de una temática. Este es uno de los resultados más importantes de esta prueba piloto. Se muestra de manera contundente que los estudiantes prefieren actividades evaluativas donde no solo se valore su nivel de conocimiento, sino que se cuente con un ambiente agradable para su realización.

Como trabajo futuro se propone darle continuidad a esta iniciativa en los cursos siguientes de la línea de Ingeniería de Software, buscando complementar las estrategias tradicionales de evaluación de tal manera que se pueda incrementar la motivación de los estudiantes y combinar juegos con aprendizaje 
significativo y desarrollo de habilidades sociales en su proceso de formación profesional.

También se propone examinar el aspecto didáctico para la aplicación de los juegos como estrategias para dinamizar el mecanismo evaluativo y aplicar un método estadístico más riguroso a partir de la información recolectada.

\section{REFERENCIAS}

[1] Ministerio de Educación Nacional - MEN. Colombia Aprende. Fecha de consulta: Febrero 2 de 2016. URL: www.colombiaa prende.edu.co

[2] A. Pérez-Gama, L. Parra-Espitia and B. PérezGutiérrez. "Education software architecture: facing student desertion in Colombia higher education with an intelligent knowledge based coaching system". In: ANDESCON. IEEE, pp. 1-5. 2010.

[3] G. López, M. Posada, C. Cardozo and D. Cuartas. "Specific actions for desertion reduction, competence identification and guidance for new students of an engineering program, a case study". In: 2nd International Congress on Engineering Education. IEEE, pp. 165-170. 2010.

[4] T. Michalko. "Thinkertoys: Handbook of creative-thinking techniques". Ten Speed Press. Berkeley, Estados Unidos, pp. 15. ISBN: 1580087736. 2010.

[5] W. Weiss. "The potential of interactive digital storytelling for the creation of educational computer games". SpringerVerlag Heidelberg. China, pp. 475-486. 2008. ISBN: 978-3-540-69734-3.

[6] I. Roffe. "Innovation and creativity in organisations: a review of the implications for training and development". Journal of European Industrial Training. Vol. 23, pp. 224-241. 1999. ISSN: 0309-0590. DOI: 10.1108/03090599910272103

[7] R.E. Mayer. "Towards a science of motivated learning in technology-supported environments". Educational Technology Research and Development. Vol. $59 \mathrm{~N}^{\circ} 2$, pp. 301-308. 2011. ISSN: 1042-1629. DOI: 10.1007/s11423-011-9188-3.

[8] A.M. Quintana, A.B. González, A. Ramos, I.R. Martín, M. Ascensión and J.
Martín. "Application of rubric in learning assessment: a proposal of application for engineering students". First International Conference on Technological Ecosystem for Enhancing Multiculturality. Salamanca, España. 2013.

[9] N. Alart. "La evaluación competencial". Aula TIC. Vol. 30, pp. 1-3. 2010.

[10] L. Ortiz. "Técnicas de evaluación". Ediciones Eco. Bogotá, Colombia, pp. 10-25. 1987.

[11] T. Morell. “¿Cómo podemos fomentar la participación en nuestras clases universitarias?". Universidad de Alicante. Marzo 1 de 2016. URL: http://hdl.handle. net/10045/13072

[12] C. Zapata and G. Giraldo. "El juego del diálogo de educción de requisitos". Avances en Sistemas e Informática. Vol. $6 \mathrm{~N}^{\circ} 1$, pp. 105-114.2009. ISSN: 1657-7663.

[13] E.M. González and M.I. Duque. "Un modelo de evaluación curricular alternativo y pertinente con el diseño curricular basado en la solución de problemas para la formación de profesionales: Caso programa de Contaduría pública de la Universidad de Antioquia". Contaduría Universidad de Antioquia. Vol. 51, pp. 105-130. 2007. ISSN: 0120-4203.

[14] E.O. Navarro, A. Baker and A. Van Der Hoek. "Teaching Software Engineering Using Simulation Games". International Conference on Simulation in Education. Milwaukee, EE.UU. 2004.

[15] E. Sweedyk and M. deLaet. "Computer games and CS education: why and how". Symposium on Computer Science Education - SIGCSE. St. Louis, EEUU. 2005.

[16] M. Staron. "Using Experiments in Software Engineering as an Auxiliary Tool for Teaching - A Qualitative Evaluation from the Perspective of Students' Learning Process". 29th International Conference on Software Engineering - ICSE. Minneapolis, EE.UU. 2007.

[17] T. Susi and M. Johannesson. Serious Games - An Overview. University of Skövde. Marzo 15 de 2016. URL: http:// www.scangame.dk/downloads/HS-IKITR-07-001_PER.pdf

[18] M. Duarte and C. Zapata. "El juego de la consistencia: Una estrategia didáctica para la ingeniería de software". Revista 
Técnica de Ingeniería Universidad de Zulia, Vol. 31, N ${ }^{\circ}$ 1, pp. 3-12. 2007.ISSN: 0254-0770.

[19] L.F. Maldonado-Granado, A.R LizcanoLlanos, E. Pineda-Ballesteros, V.A. Uribe-Otálora and J.B. Sequeda-Tarazona. "Comunidades de aprendizaje mediadas por redes informáticas". Educación y Educadores. Vol. $11 \mathrm{~N}^{\circ} 1$, pp. 199-224. 2008. ISSN: 0123-1294.
[20] L. Recio and P. Montero. "Una experiencia de aprendizaje universitario mediante juegos de torneo en clase". REDU. Revista de Docencia Universitaria. Vol. 12, pp. 415-436. 2016.

[21] K.Z. Watkins. "Peer evaluation as a needed web 2.0 activity in project management for teaching practical software engineering". 10th ACM Conference on SIG-Information Technology Education. Fairfax, Virginia, USA, ACM. 2009. 